Mens

Revue d'histoire intellectuelle et culturelle

mens

\title{
Enjeux culturels et sociaux de la circulation de la chanson J'attendrai dans les années 1940
}

\section{Simon-Pierre Beaudet et Chantal Savoie}

Volume 12, numéro 2, printemps 2012

Enjeux et modalités des transferts culturels dans la vie artistique canadienne-française de la première moitié $d u \mathrm{XX}^{\mathrm{e}}$ siècle

URI : https://id.erudit.org/iderudit/1013873ar

DOI : https://doi.org/10.7202/1013873ar

Aller au sommaire du numéro

Éditeur(s)

Centre de recherche en civilisation canadienne-française

ISSN

1492-8647 (imprimé)

1927-9299 (numérique)

Découvrir la revue

Citer cet article

Beaudet, S.-P. \& Savoie, C. (2012). Enjeux culturels et sociaux de la circulation de la chanson J'attendrai dans les années 1940. Mens, 12(2), 37-59.

https://doi.org/10.7202/1013873ar

\section{Résumé de l'article}

Cette analyse aborde le cas singulier de la circulation de la chanson J'attendrai dans la culture des années 1940, dont l'analyse des variantes permet de cerner les rapports mouvants d'une chanson aux genres chansonniers et musicaux, aux goûts et aux pratiques du public, ainsi qu'à leur ancrage au sein des champs culturels nationaux. Elle permet même, parfois, de sonder les modalités de l'évolution des modèles des rapports sociaux entre les sexes présents dans différentes versions. Ce sont ainsi autant les enjeux artistiques, culturels, sociaux et intimes à l'oeuvre qui guident notre analyse de ce cas de transfert culturel, que le repérage de vecteurs permettant de formuler une équation de la circulation culturelle des chansons à succès. 


\title{
Enjeux culturels et sociaux de la circulation de la chanson J'attendrai dans les années 1940
}

\author{
Simon-Pierre Beaudet \\ Département des littératures \\ Université Laval \\ Chantal Savoie \\ Département d'études littéraires \\ Université du Québec à Montréal
}

\section{Résumé}

Cette analyse aborde le cas singulier de la circulation de la chanson J'attendrai dans la culture des années 1940, dont l'analyse des variantes permet de cerner les rapports mouvants d'une chanson aux genres chansonniers et musicaux, aux goûts et aux pratiques du public, ainsi qu'à leur ancrage au sein des champs culturels nationaux. Elle permet même, parfois, de sonder les modalités de l'évolution des modèles des rapports sociaux entre les sexes présents dans différentes versions. Ce sont ainsi autant les enjeux artistiques, culturels, sociaux et intimes à l'œuvre qui guident notre analyse de ce cas de transfert culturel, que le repérage de vecteurs permettant de formuler une équation de la circulation culturelle des chansons à succès.

\section{Abstract}

This article deals with the singular case of the circulation of the song J'attendrai in the 1940s. The analysis of the different versions of the song allows us to reveal the changing relationship between these versions and musical genres, the tastes and practices of the public, and the way Mens, vol. XII, $\mathrm{n}^{\circ} 2$ (printemps 2012) 
they fit into national cultures. This analysis also allows us, in places, to explore the evolution of social relationships between genders as presented in the different versions of the song. This way, our analysis of this case of cultural transfer is guided as much by the artistic, cultural, social and intimate aspects of the song, as by a search for the vectors explaining the cultural circulation of a successful song.

Entrepris en 2006, nos travaux sur « Les préférences musicales des lectrices du Bulletin des agriculteurs dans les années 1940 $»$ ont pour objectif d'aborder le phénomène de la chanson populaire des années 1940 du point de vue de sa réception par le grand public, et de tenter de cerner les modalités du succès en tenant compte du contexte socioculturel au sein duquel il se manifeste ${ }^{2}$. Cette recherche a permis de reconstituer un palmarès des chansons les plus appréciées du public féminin rural au cours des années 1940; le corpus des 28000 "demandes " que nous avons compilées a permis de répertorier les 70 chansons les plus "demandées", chansons pour lesquelles nous avons constitué une fiche de renseignements, recensé les différentes versions ayant circulé au cours des années 1940, rassemblé bon nombre d'enregistrements et, enfin, entrepris des analyses internes afin d'en dégager les principales caractéristiques structurelles, thématiques, sonores, etc. La considération de l'ensemble de ces données, malgré le fait que les travaux ne soient pas complètement achevés à ce jour, permet de constater que cette saisie de la chanson populaire par la lorgnette du goût féminin rural au cours de la décennie 1940 offre une vue qui tranche résolument avec la connaissance synthétique véhiculée dans la majorité des sources secondes

\footnotetext{
${ }^{1}$ Nous sommes reconnaissants du soutien financier que nous avons reçu du Fonds de recherche du Québec - Société et Culture, programme Établissement de nouveaux chercheurs.

2 Pour un aperçu des objectifs et de la méthode de l'ensemble de cette recherche, voir Chantal Savoie, "Les préférences musicales des lectrices du Bulletin des agriculteurs, 1939-1955 [Note de recherche] ", Communication, vol. 25, $\mathrm{n}^{\circ} 1$ (automne 2006), p. 258-265.
} 
servant à faire l'histoire de la chanson de cette époque ${ }^{3}$. En effet, les sources secondes usuelles tendent à souligner la co-présence de plusieurs répertoires, notamment du "folklore " de La Bonne Chanson, de la chansonnette d'origine française, des Variétés lyriques et du répertoire western en émergence, et à privilégier l'angle des influences étrangères, françaises ou américaines. Ces influences sont jugées néfastes, dans la mesure où elles sont perçues comme pouvant constituer une menace aux valeurs traditionnelles de la société canadienne, soit en favorisant l'assimilation des Canadiens français par des contacts plus étroits avec la culture américaine abondamment diffusée par la radio, soit en diffusant des modèles culturels jugés immoraux, comme ceux que présente la chanson sentimentale française. Sur le plan socioesthétique de l'histoire de la chanson plus proprement canadienne-française, le récit proposé par la majorité des sources tend alors à décrire la chanson populaire du Québec des années 1940 comme révélatrice d'une période de transition au sein de laquelle la production chansonnière nationale "de qualité " jouerait un rôle marginal, du moins lorsqu'on la mesure à l'aune des années 1950,1960 et $1970^{4}$.

${ }^{3}$ Mentionnons à titre d'exemples, et sans prétendre ici à l'exhaustivité, les ouvrages de Robert Léger (La chanson québécoise en question, Montréal, Québec Amérique, 2003), Bruno Roy (Pouvoir chanter, Montréal, VLB éditeur, 1991; Et cette Amérique chante en québécois, Montréal, Leméac, 1978; Panorama de la chanson québécoise, Montréal, Leméac, 1977), Benoit L’Herbier (La chanson québécoise, Montréal, Les Éditions de l'Homme, 1974), Jean-Nicolas De Surmont (La Bonne Chanson : le commerce de la tradition en France et au Québec dans la première moitié du XXe siècle, Montréal, Triptyque, 1910), Roger Chamberland et André Gaulin (La chanson québécoise de la Bolduc à aujourd'hui, Québec, Nuit blanche éditeur, 1994). Soulignons par ailleurs les perspectives plus nuancées de la synthèse proposée par Danielle Tremblay («La chanson québécoise et ses cousines », [En ligne], [http:// www.chansonduquebec.com/] (24 avril 2012)), de même que l'article de Renée Berthe Drapeau "Chant, chanson et chansonnette au Québec (1920-1950)" (dans Robert Giroux (dir.), La chanson dans tous ses états, Montréal, Triptyque, 1987, p. 163-186).

4 Pour le reste, nous avons surtout accès aux discographies et aux sources qui mettent en valeur la production culturelle, de même qu'à des bilans de l'émergence de la radio, de son importance dans la diffusion de la chanson (principales émissions, 
Les résultats de nos recherches en cours sur la réception de la chanson populaire à cette époque, considérés dans leur globalité, incitent, au-delà de l'hétérogénéité des artistes et des styles musicaux représentés, à nuancer ces perspectives ${ }^{5}$. Trois constats généraux s'en dégagent. En premier lieu, les chansons populaires interprétées en français dominaient très largement les préférences musicales populaires des femmes dans les années 1940 (alors que le "péril culturel » qu'auraient constitué les ondes radiophoniques saturées de chansons américaines est un lieu commun persistant dans la majorité des sources secondes citées). En deuxième lieu, les versions locales de chansons françaises ou américaines jouaient un rôle important dans la circulation des chansons les plus populaires; ces versions locales constituent, en outre, un indicateur de succès souvent négligé et jouent un rôle d'embrayeur au sein d'une industrie locale de la popularité médiatique en pleine effervescence pendant la Seconde Guerre mondiale. Troisièmement, les chansons les plus demandées, peu importe le style musical auquel elles appartiennent ${ }^{6}$, confirment la prépondérance du sentiment et de la quête amoureuse comme thème dominant du corpus. Toutefois, force est de constater, après l'analyse plus détaillée d'une dizaine de titres, que ces premiers résultats de notre travail permettent avant tout de formuler avec plus d'acuité plusieurs questions que nous avions au départ, dont un bon nombre d'entre

artistes et animateurs vedettes, etc.) et des ajustements structurels que ce nouveau média provoque dans l'industrie du disque.

5 D'autres travaux contribuent significativement à cette relecture. Soulignons notamment les mémoires et les thèses de Sandria Bouliane (L'impact de Herbert Berliner et Roméo Beaudry sur la structuration du champ de la phonographie populaire canadienne-française, 1918-1932, mémoire de maîtrise, Québec, Université Laval, 2006), de Catherine Lefrançois (La chanson country-western, 1942-1957, thèse de doctorat, Québec, Université Laval, 2011) et de Luc Bellemare (Le style dans les chansons enregistrées de Félix Leclerc : une analyse des relations texte-guitare, mémoire de maîtrise, Québec, Université Laval, 2007).

6 Soulignons, sans entrer dans les détails ici, que le style musical, du point de vue des goûts musicaux à tout le moins, est souvent associé à une " mode ", et que l'époque est marquée par une succession de plus en plus rapide de ces modes, caractéristique d'une fuite en avant, typique d'une certaine modernité médiatique et culturelle. 
elles concernent différents aspects de la circulation des biens culturels, les effets de ces circulations géographiques, musicales et médiatiques simultanées et entrecroisées et, enfin, les pratiques qui les accompagnent et les rendent signifiantes dans différents contextes.

En effet, quelles orientations ces résultats offrent-ils si l'on intègre la faveur du public à l'histoire de la chanson? Comment cette histoire de la chanson populaire modulée par la prise en compte des usages contribue-t-elle à une meilleure compréhension tant des pratiques sociales que musicales, incluant les pratiques d'écoute et la façon dont elles s'insèrent dans le mode de vie? Parmi les pistes qui s'annoncent fertiles, la question de la circulation des chansons et des différents processus de médiation et d'appropriation auxquels elles donnent lieu occupe une place prépondérante. Afin d'amorcer ce volet de notre réflexion, nous avons entrepris l'analyse d'un cas singulier, qu'on peut considérer comme emblématique sur le plan de la circulation et des transferts culturels, celui de la chanson J'attendrai. Ce titre se démarque, d'une part, par le nombre important de versions locales et étrangères qu'il a suscité avant les années 1950 et que nous avons pu repérer. Il se révèle, d'autre part, riche sur le plan des variantes et, plus précisément, des liens que ces variantes (structurelles, musicales, textuelles, stylistiques, etc.) peuvent entretenir avec les différents contextes culturels et les aires géographiques où circulent la chanson. Nous proposons donc ici un survol historique des versions et des variantes de J'attendrai, avant de nous attarder à quelques aspects des transferts culturels que révèle l'histoire protéiforme de cette chanson et qui nous semblent enrichir notre compréhension des enjeux soulevés par la circulation des chansons et, plus largement, sur la notion de succès telle qu'elle se manifeste au cours de la décennie qui nous intéresse.

\section{La chanson et ses versions}

Les données compilées pour notre étude inscrivent la chanson J'attendrai au troisième rang parmi les chansons les plus demandées 
au cours de l'année 1940. Le Bulletin des agriculteurs, créé en 1918, est avant tout un périodique destiné aux gens des métiers agricoles, mais il instaure, au sortir de la crise économique de 1929, des pratiques qui le distinguent et contribuent fortement à son succès, notamment en misant sur les stratégies éditoriales des magazines. C'est dans ce contexte qu'on crée la section "Votre domaine, mesdames ", destinée aux femmes de la famille, alors convoitées autant pour accroître le nombre d'abonnements que pour la publicité que la conquête de ce nouveau public permet de vendre aux annonceurs et qui élargit considérablement la palette des produits annoncés dans le Bulletin. Cette section féminine compte à ce moment pour près de la moitié du périodique, et sa présence accompagne l'importante croissance du lectorat du Bulletin durant les années 1930 et 1940 (de 12000 abonnés à la fin des années 1920, le Bulletin passe à 60000 abonnés à la fin des années 1930 et à plus de 140000 à la fin des années 1940) ${ }^{7}$. Le contenu de ces pages féminines s'apparente, dans son ensemble, à celui des périodiques féminins de l'époque, dans lesquels on trouve des conseils pratiques, de la cuisine, de la mode, quelques récits et, bien sûr, de la publicité vantant les mérites de tout produit qu'une bonne maîtresse de maison pourrait vouloir se procurer. Rappelons que la nature particulière de notre source, soit les demandes de chansons formulées par les femmes dans une page de correspondance de la section féminine du Bulletin ${ }^{8}$, ne nous permet pas d'associer clairement un

7 On consultera avec profit l'étude de Suzanne Nolin (Histoire, littérature et subversion dans le Bulletin des agriculteurs, 1940-1959, mémoire de maîtrise, Université de Sherbrooke, 2003), qui porte sur le contenu littéraire et culturel du Bulletin.

${ }^{8}$ Chaque demande consiste en la mention du titre d'une chanson, du nom de la femme qui fait la demande et de la désignation de sa ville de résidence. Les demandes sont alignées les unes à la suite des autres et présentées en colonnes. Le nombre de demandes par édition varie de quelques dizaines à plusieurs centaines, selon la popularité de la pratique. Ces demandes coexistent avec plusieurs autres, tant dans les périodiques qu'à la radio. À partir du deuxième tiers des années 1940, les demandes semblent surtout reléguées aux ondes radiophoniques et ont laissé moins de traces. Certains témoignages d'artisans du monde de la radio dans 
titre et son interprète ${ }^{9}$, et que c'est dans ce contexte que nous avons dès le départ voulu tenir compte d'un ensemble de versions contemporaines pour chaque chanson. Demandée 115 fois en 1940, la chanson J'attendrai côtoie au palmarès Le bonheur est entré dans mon cour et Le bateau des îles, toutes deux également enregistrées par différents interprètes en France et au Québec.

Écrite en 1933 par Nino Rastelli (texte original en italien ${ }^{10}$ ) et Dino Olivieri (musique) sous le titre Tornerai, J'attendrai est une chanson d'amour à résonance intimiste, exploitant le thème de l'amour qui dure malgré le passage du temps. De manière générale, la simplicité de l'imagerie laisse peu d'écart avec son niveau référentiel, permettant une saisie immédiate du sens, lequel est soutenu par une mélodie languissante et la répétition du "j'attendrai », en plus de la reprise du refrain. La chanson est structurée sur l'opposition d'un temps présent incarné dans des images cycliques qui évoquent l'attente (rythme cardiaque, horloge, jours, saisons, etc.) et d'un temps futur qui éternise à jamais cette attente, notamment en refoulant constamment le moment du retour de l'être aimé hors des limites des trois minutes de la chanson. Ce temps futur, celui du refrain, est concrétisé par quelques éléments temporels évoquant la linéarité cette fois, comme l'irruption d'indices sonores (bruits lointains) qui viennent rompre le cycle du présent, aviver l'espoir du retour et entretenir la ferveur de l'attente proférée de manière itérative. Le présent de l'attente, qui se manifeste, pour sa part, dans les couplets, est renforcé par des images spatiales associées à la maison (le feu de l'âtre, la porte, le jardin, le nid), auxquelles s'oppose le déplacement

les années 1930 et 1940 restent une source précieuse dans le domaine. Voir à ce sujet Jean Du Berger, Jacques Mathieu et Martine Roberge, La radio à Québec, 1920-1960, Québec, Les Presses de l'Université Laval, 1997.

9 Rappelons que l'on demande les chansons par titre et qu'on ne mentionne que très exceptionnellement l'interprète. Il nous semble très imprudent d'agir autrement qu'en considérant l'ensemble des versions au cours d'une même période, le nombre de versions étant lui-même un indicateur du succès.

${ }^{10}$ La version française est de Louis Poterat. 
(s'enfuit, retour) de l'absent-e. Ce récit très dépouillé circule sous toutes sortes de formes, qui en font parfois varier le sens et la portée, et l'histoire de sa circulation constitue une topographie des méandres d'un succès et des récits qu'il porte et qui le portent.

\section{Sur scène}

La chanson est portée par une mélodie accrocheuse, inspirée d'un air sans paroles tiré de l'opéra Madame Butterfly de Puccini. Rappelons-en brièvement l'intrigue : officier américain de passage à Nagasaki, B.F. Pinkerton épouse une jeune geisha mais, contrairement à cette dernière, il ne prend guère ce mariage au sérieux. Pinkerton ayant été rappelé par son service, Cio-cio-San («Madame Butterfly ") espère le retour de son époux et élève l'enfant issu de leur nuit de noces, tout en refusant de nombreuses propositions de mariage. Après une absence de trois ans, Pinkerton revient avec une épouse américaine. Leur laissant l'enfant, Butterfly meurt dans l'honneur en se poignardant. L'air dont la musique d'Olivieri s'inspire est le "Coro a bocca chiusa" ", qui survient à la fin du deuxième acte, précisément au moment où Butterfly attend que Pinkerton descende du bateau au moment de son retour.

\section{Au cinéma}

À sa création en 1904, l'opéra de Puccini connaît, après des débuts difficiles, un succès international, et l'œuvre est adaptée à trois reprises au cinéma avant l'écriture de J'attendrai. Dès 1915, Sydney Olcott produit Madame Butterfly avec Mary Pickford, aussi fidèlement que possible. Forbidden City, en 1918, qui reprend les grandes lignes narratives de l'œuvre en déplaçant l'action en Chine, et The Toll of the Sea, film réalisé en Technicolor en 1922 par Chester M. Franklin, reprennent tous les deux la trame narrative de l'opéra, tout en faisant preuve d'une certaine liberté. Plus significative est

${ }^{11}$ Littéralement, chœur à bouche fermée. 
l'adaptation de David Belasco en 1932, mettant en vedette Cary Grant et Sylvia Sydney. La musique du film est adaptée par W. Franke Harling de l'opéra de Puccini et présente également ce que les Américains appellent le "Humming Chorus", le "Coro a bocca chiusa", qui deviendra l'air de J'attendrai.

Ainsi, J'attendrai est écrite et paraît quelque trente ans après la création de l'opéra de Puccini, qui fut diffusé autant sur les scènes d'opéra qu'au cinéma. Tout laisse donc croire que la chanson a ainsi circulé en étant associée à l'idée d'un amour durable par-delà l'épreuve du temps, avec un rappel plus ou moins direct ou conscient, chez le spectateur, du drame de Butterfly, particulièrement dans ses versions européennes et états-uniennes. Les paroles génériques y contribuent, dans la mesure où le texte aurait bien pu s'insérer dans l'opéra de Puccini sans jurer outre mesure.

\section{La trace des partitions}

L'histoire de la circulation de la chanson J'attendrai passe également, bien sûr, par la scène des variétés ${ }^{12}$ et par la circulation des différentes éditions des partitions. Sans prétendre en suivre la trace de manière exhaustive puisque les dates de notre corpus correspondent avant tout à celles d'un noyau important d'enregistrements sonores auquel nous reviendrons plus loin, nous avons été attentifs aux partitions éditées avant les enregistrements sonores qui ont fixé cet air, voire en ont fait un "standard ", ou un classique, comme l'enregistrement de Rina Ketty en 1938. Ce sont ainsi surtout les partitions italiennes qui permettent de suivre la trace de Tornerai, puisque des partitions

${ }^{12}$ La circulation de cette chanson devrait pouvoir être retracée par des recherches concernant différents agents littéraires très actifs à l'époque, et qui négociaient des ententes de droits d'auteurs, tout en stimulant l'organisation et la production de spectacles par la publication de Guides. Nous remercions Jean-Pierre Chalifoux de nous avoir fourni de précieux renseignements. 
musicales sont publiées en $1927^{13}, 1930^{14}$ et $1936^{15}$. L'air paraît donc circuler en se modelant à différents styles et versions, le tango et la valse notamment. C'est toutefois la version éditée par Olivieri et Rastelli en 1936 qui semble fixer l'univers sonore avec la plus grande durabilité. Les partitions italiennes le désignent désormais comme un "slow ", et c'est sous cette forme que la chanson joue le rôle d'embrayeur pour les enregistrements sonores qui suivent.

\section{Sur disque}

Même si tous les méandres de la circulation de la chanson n'ont pu être retracés, nous avons néanmoins constaté avec intérêt que la pièce est enregistrée pour la première fois en français à Montréal en 1934, sur étiquette RCA Victor, par la chanteuse d'origine française Paulette Mauve, sur des paroles françaises de Louis Poterat ${ }^{16}$. Paulette Mauve est accompagnée sur cet enregistrement par l'organiste Léo Le Sieur, qui travaillera notamment avec Lucille Dumont et Monique Leyrac. Pour curieuse qu'elle paraisse, cette première version en sol québécois, qui peut suggérer des liens concrets méconnus entre des acteurs particuliers, notamment des agents qui représentent les artistes et des éditeurs de musique, n'est pas unique, comme nous le verrons plus loin, et semble indiquer qu'on gagnerait à reconstituer plus systématiquement le réseau des échanges dans le domaine de la chanson populaire.

La version " originale » en italien aurait, pour sa part, été enregistrée pour la première fois en 1937 par le trio Lescano, composé de trois sœurs d'origine hongroise aux noms italianisés. La version

13 Tornerai ... Tango [per pianoforete canto] versi di Nando Vitali, à Florence, chez Saporetti e Cappelli, en 1927, 5 p. Pour toutes ces références tirées du catalogue collectif des bibliothèques italiennes, nous sommes redevables à M. Jean-Pierre Chalifoux.

14 Tornerai : intermezzo-valse [per orchestrina], parue à Turin, chez L. Checchini, en 1930, 8 p.

15 Dino Olivieri, Tornerai; slow /parole di Nino Rastelli, Novara, P. Leonardi, 1936, rééditée en 1937 et 1938.

16 Parolier français (Troyes, 1901 - Genève, 1982). 
des Lescano est axée sur les harmonies vocales - on les présente comme une " réponse italienne " à des groupes vocaux comme les Andrew Sisters. Présentées comme un trio «swing / jazz », les chanteuses sont effectivement accompagnées par une orchestration au rythme plus appuyé, où la ligne mélodique est donnée par une clarinette à l'avantplan. Indice du succès de la chanson, Carlo Buti ${ }^{17}$ donne sa propre version en italien quelques mois plus tard, au cours de l'année 1937.

C'est pourtant en France que J'attendrai connaîtra un succès populaire qui assurera son rayonnement international, relançant une nouvelle vague de circulation, accélérée, de la chanson. Rina Ketty ${ }^{18}$ se fait connaitre par sa version donnée en 1938, et sa renommée, à l'époque, doit beaucoup à ce titre. Encore ici, le succès se confirme quand Tino Rossi la reprend peu après. Django Reinhardt et Stéphane Grappelli en offrent une version instrumentale la même année, témoignant d'un processus de standardisation qui commence à transcender les styles musicaux. Les versions subséquentes sont nombreuses, quoique moins significatives dans le cadre de notre recherche.

Toujours en 1938, aux États-Unis, les droits de la chanson sont enregistrés par Anna Sosenko pour l'éditeur Southern sous le titre I'll Be Yours. La chanteuse se produit essentiellement dans les cabarets et les music-halls, ce qui nous laisse croire que la pièce a pu connaître beaucoup de succès à la scène avant son enregistrement pour Decca en $1945^{19}$, que Billboard recense la même année. Mentionnons que

17 Le ténor italien Carlo Buti (1902-1963), surnommé le Bing Crosby italien, est considéré comme le maître de la sérénade. Il est célèbre pour ses ballades sentimentales et aurait enregistré plus de 1500 chansons au cours de sa carrière, en plus d'être la vedette de plusieurs films.

18 Née Cesarina Picchetto (Sarzane, Italie, 1911 - Cannes, 1996).

19 Rappelons que les années 1930 et 1940 constituent une période de transition entre les chansons diffusées essentiellement sur scène et en tournée, et celles qui circuleront bientôt massivement grâce aux nouveaux moyens technologiques que sont la radio et le disque, désormais bien implantés et devenus des locomotives de l'industrie. Voir à ce sujet l'ouvrage de Gérôme Guibert, La production de la culture : le cas des musiques amplifiées en France, Paris, Mélanie Seteun/Irma, 2006. 
circule également une partition anglaise titrée Au revoir (J'attendrai), sur un texte de Bruce Sievier. Bing Crosby la reprendra également.

La fortune de la chanson, après cette première série de versions produites dans les années 1930 et 1940, persiste. L'air traverse encore une fois plusieurs styles musicaux : on en recense des versions pop à la Sinatra (Frankie Avalon, en anglais), hawaïenne (Roland Peachey and His Royal Hawaiian, chantée en français), voire disco (Dalida), d'autres versions en allemand et en tchèque, sans compter celles qui ont échappé à notre recherche. On semble être ici au cœur d'un processus de classicisation ${ }^{20}$ : passée dans le domaine public, la chanson se trouve ponctuellement réactualisée par chaque nouvelle version, tout en mobilisant à divers degrés le souvenir des anciennes. D'abord reçue, dans le sillage de l'opéra de Puccini, comme une icône de l'amour qui dure par-delà l'épreuve du temps, particulièrement dans ses versions européennes et états-uniennes, la chanson connaîtra un nouveau type de réception avec l'arrivée de la Seconde Guerre mondiale, alors que le référent spontané qui conditionne la lecture de la pièce deviendra celui d'une femme qui attend son mari ou son amoureux parti à la guerre ou, plus généralement, dans l'attente de la fin du conflit. La pièce, par sa référence au présent de l'attente et au futur toujours reporté, peut également être associée au passé, et tout particulièrement à un passé heureux, celui d'avant l'attente, la séparation ou l'élément perturbateur quel qu'il soit. Qui plus est, pour un public de plus en plus éloigné du moment de sa création, la chanson évoque l'époque des parents ou des grands-parents. Elle en vient ainsi à s'apparenter à un hymne ${ }^{21}$, à une période révolue dont on souhaite revivre la sérénité et la plénitude dans le futur et

${ }^{20}$ Nous entendons par processus de classicisation les différentes étapes qui assurent la légitimité, puis la mémoire des œuvres culturelles marquantes dans une société donnée. Alain Viala, dans son article "Qu'est-ce qu'un classique " (Bulletin des bibliothèques de France, $n^{\circ} 1$ (1992), p. 6-15), en livre une éclairante synthèse.

21 À propos des diverses modalités du processus d'hymnodisation, voir les travaux de Jacques Cheyronnaud et notamment son article « La parole en chantant : musique et cultures politiques ", Protée, vol. 30, n 1 (2002), p. 79-94. 
devient l'incarnation même de la nostalgie. Le cinéma, passé et présent, contribue pour sa part largement à sémantiser la chanson comme un hymne à la fin de la guerre, bien que les reprises récentes au Québec, notamment lors de la campagne publicitaire Nostalgie, réalisée par l'agence BBDO pour le compte de la Fédération des producteurs de lait du Québec 22 à la fin des années 1990, exploitent les connotations nostalgiques reliées à l'univers maternel et au monde de l'enfance. La chanson évoque alors « l'ancien temps » des parents et des grands-parents comme une sorte de paradis perdu, et son succès suggère une relecture pertinente et efficace de la chanson pour différentes générations.

\section{Circulation géographique et transferts culturels}

S’il est parfois difficile de retracer la chronologie des différentes versions, ce tour d'horizon permet au moins de saisir la rapidité surprenante avec laquelle les chansons sont diffusées et les multiples formes sous lesquelles se fait cette circulation qui est tout, sauf linéaire. Il convient, bien sûr, de replacer la circulation de la chanson dans le modèle qui prévaut à l'époque au sein de la culture et de l'industrie de la chanson au Québec. Des chansons américaines circulent au Québec et certaines sont traduites et enregistrées ici; la même chose vaut pour les chansons françaises : certaines versions passent directement en contexte québécois, d'autres sont adaptées et/ou réenregistrées par des interprètes locaux. Certaines sont même soumises à une forme de " commerce triangulaire » dans le cas de

22 Luc Dupont, professeur au Département de communication de l'Université d'Ottawa fait une brève synthèse de l'histoire des publicités du lait au Québec. Voir « Comment expliquer le succès des pubs de lait ", dans son carnet en ligne, ([http://lucdupont.blogspot.ca/2010/10/comment-expliquer-le-succes-des-pubsdu.html]) (27 avril 2012). Il y mentionne notamment que : « Fait plutôt unique dans l'histoire de la pub au Québec, les messages de la campagne Nostalgie généreront un taux de satisfaction de $98 \%$ ! Dans la foulée des messages TV, un CD promotionnel mettant en vedette Bécaud, Trenet et Salvatore Adamo (L'Album blanc) vendra plus de 200000 copies. " 
chansons américaines, traduites et publiées en France et, encore une fois, adaptées au Québec ${ }^{23}$. Chaque fois, divers processus de médiation sont en jeu : le style musical, les arrangements et l'ensemble de l'univers sonore, l'interprétation. Le texte également présente des différences, et pas uniquement pour ce qui concerne les traductions puisque, parfois, on semble adapter le texte en fonction de critères qui tiennent autant à la production industrielle et artistique qu'au public visé, par exemple en insérant ou en enlevant un intermède musical, en ajoutant ou en omettant un couplet, en doublant un refrain, altérant parfois la structure couplet/refrain de manière importante. Dans le cas qui nous occupe, le processus paraît encore plus singulier, du moins dans la mesure où la carrière de la chanson dans sa version en langue française commence vraisemblablement au Québec, par l'entremise d'une chanteuse française établie à Montréal, et se poursuit en Europe et aux États-Unis, comme nous l'évoquions au début de cet article.

Parmi ce vaste ensemble de versions et au sein des méandres de leur circulation, deux versions québécoises de J'attendrai retiennent tout particulièrement notre attention pour l'instant puisqu'elles présentent les cas les plus denses sur le plan du nombre et des types de transferts à l'œuvre. Ce sont en outre celles qui, à notre avis, correspondent le mieux, sur le plan synchronique, aux données du Bulletin des agriculteurs que nous avons compilées et qui témoignent, de ce fait, de l'importance du succès de la chanson J'attendrai auprès d'un public féminin de différentes régions du Québec en 1940. Enregistrées par Ludovic Huot et Jean Lalonde en 1938, la même année que les versions de Ketty et de Rossi, la chanson fait ainsi l'objet de réappropriations québécoises par deux interprètes différents, quelques années après l'enregistrement de Paulette Mauve. Ludovic

${ }^{23}$ Voir Serge Lacasse et Chantal Savoie, "De "Rendez-moi mes montagnes" à "Loin des guitares" : la chanson d'ici sous influence ", dans Yvan Lamonde et Denis Saint-Jacques (dir.), 1937 : un tournant culturel, Québec, Les Presses de l'Université Laval, 2009, coll. «Cultures québécoises », p. 161-171. 
Huot, des Variétés lyriques, accompagné par l'orchestre McIver, l'enregistre pour l'étiquette Starr. Le même orchestre accompagne également Jean Lalonde, chanteur de charme et vedette de la radio, qui donne sa version pour les disques Bluebird.

La contemporanéité et la coexistence de ces deux versions québécoises avec le noyau de production des versions en 1938 n'étonne pas outre mesure, le phénomène étant typique du modèle de circulation de la chanson populaire à l'époque, comme nous l'avons constaté, et à différentes échelles, pour de nombreuses chansons de cette période. Nous trouverions très probablement le même genre de versions locales dans nombre de pays d'Europe de l'Ouest à la même époque. En outre, la tournée de Tino Rossi au Québec la même année est reconnue pour avoir suscité de nombreuses vocations de chanteurs de charme ${ }^{24}$. Mais nous intéresse davantage ici le jeu des variantes qui surgissent lors de la comparaison des différentes versions, puisque ces variantes, certaines d'entre elles notamment, nous ont semblé constituer des traces de médiation et de transferts culturels à l'œuvre au cours d'un processus qui s'amorce par la circulation de la chanson. En effet, et en premier lieu, la structure de la chanson diffère d'une version à l'autre, départageant assez nettement les versions québécoises et françaises ${ }^{25}$. On retrouve ainsi deux grandes familles de versions : celle qui commence par le refrain (les versions françaises, celles de Jean Sablon, de Rina Ketty et de Tino Rossi) et celle qui commence par un couplet (les versions enregistrées au Québec, celles de Paulette Mauve, de Jean Lalonde et de Ludovic Huot). Nous avons d'abord tenté de vérifier si ces différences pouvaient être attribuables à des standards différents, et notamment à une influence grandissante de la musique populaire américaine et de la structure associée aux chansons de la Tin Pan

${ }^{24}$ Voir Drapeau, "Chant, chanson et chansonnette au Québec (1920-1950)».

25 Il existe plusieurs autres différences entre les versions, dont la présence ou l'absence d'un couplet dans certaines versions. Toutefois, sur le plan des transferts culturels, cette distinction nous a semblé peu porteuse. Quant à l'exégèse des innombrables variantes, nous ne la détaillons pas ici, car cela nous éloignerait de notre propos. 
Alley $^{26}$. Toutefois, l'existence de versions américaines, dont celle de Bing Crosby qui commence elle aussi par le refrain, érode sérieusement la pertinence de cette hypothèse qui tendait à associer la structure de la chanson aux modèles culturels et esthétiques de la production de la musique enregistrée. D'ailleurs, à ce jour, toutes les versions de la chanson que nous avons pu écouter, à l'exception des versions québécoises, commencent par le refrain.

Outre l'ordre des sections de la chanson, l'autre élément qui semble distinguer sensiblement les versions québécoises des autres tient à une certaine légèreté de l'interprétation, un détachement qui se dégage de l'éthos de l'interprète (voix moins aiguës, trémolo plus discret, rythme moins lent et langoureux, etc.), et qui tend ainsi à diminuer l'impact de l'émoi et de la passion amoureuse suggérés par les autres interprétations. En plus de ces caractéristiques liées à l'interprétation, on remarque que le rythme et les orchestrations des versions québécoises de J'attendrai, s'ils invitent à la danse, évitent cependant de faire de la chanson un slow et relèvent davantage du swing, voire du ragtime. Pourrait-on inférer que ces versions, dont les caractéristiques vocales et rythmiques sont moins émotives et suggestives, auraient davantage le potentiel d'amadouer certains réfractaires aux musiques étrangères, perçues comme dangereuses pour les bonnes mœurs? Rappelons qu'à cette époque, les journaux plus conservateurs regorgent d'attaques contre la chanson sentimentale, dont on dénonce les allusions à des mœurs légères, au profit du folklore dont le propos est jugé plus sain. S'oppose, en silence, à ce discours péremptoire, des périodiques plus légers qui, sans faire l'apologie de la chanson sentimentale populaire, exploitent la popu-

26 Rappelons que cette expression sert à désigner l'âge d'or de la musique populaire américaine au tournant du $\mathrm{Xx}^{\mathrm{e}}$ siècle, et fait référence au nom de la rue où s'étaient rassemblés les plus importants éditeurs de musique en feuilles. Le nom a ensuite été associé au style de musique qui a constitué la signature de cette époque, et dont la structure est caractéristique. Pour une description d'ensemble, voir la notice "Tin Pan Alley » signée par Mark Tucker et Peter Gammond dans l'encyclopédie Oxford Music On-line, [http://www.oxfordmusiconline.com]. 
larité des vedettes en général et l'engouement des jeunes filles pour les interprètes masculins de ce genre ${ }^{27}$. Dans ce contexte, l'hypothèse d'un lien entre la facture sonore et l'érosion du potentiel jugé subversif des chansons semble simpliste, mais il serait malhonnête de ne pas lui accorder un peu de plausibilité.

Il est intéressant de constater que la particularité des versions québécoises se maintient en dépit de la « pression » de la standardisation qu'aurait pu exercer la diffusion des versions françaises, et tout particulièrement celles de Rina Ketty et de Tino Rossi, qui sont devenues la référence, même en Italie, semble-t-il. Outre ces quelques remarques à propos des rapports qu'il est parfois possible d'esquisser entre la facture d'une chanson et sa circulation dans différentes aires géographiques mais surtout culturelles, d'autres aspects liés à la circulation de J'attendrai nous paraissent intéressants à considérer, dans la mesure où ils donnent des indices sur des chemins moins fréquentés dont la prise en compte enrichit l'histoire de la chanson populaire en la rendant plus concrète et plus complexe, tout en favorisant la formulation de synthèses moins réductrices. Nous sommes ici devant un cas rencontré au moins à deux autres reprises parmi nos chansons les plus demandées par les lectrices du Bulletin des agriculteurs: celui où des chansons italiennes sont d'abord adaptées au Québec avant d'être reprises en France. C'est le cas de Per un bacio d'amor, enregistrée par Carlo Buti en 1939, reprise en 1940 par Lucille Dumont et que Tino Rossi reprend à son tour en 1953. Toujours en 1939, La Piccina du même Carlo Buti devient au Québec L'armée et la marine en 1940 et, la même année, est enregistrée aux États-Unis par les Andrew Sisters, sous le titre Ferry Boat Serenade. Ce lien avec les chansons italiennes - et particulièrement avec l'interprète Carlo Buti qui revient dans toutes les occurrences - mérite

27 Voir à ce sujet le résumé de la communication de Chantal Savoie, intitulée "Chanson imprimée et chanson chantée dans les années 1940 » et présentée lors de la Journée d'échanges de l'Association québécoise pour l'étude de l'imprimé (AQÉI) qui s'est tenue le 13 avril à l'Université McGill, dans le Bulletin de l'AQÉI, $n^{\circ} 44$ (automne 2012). À paraître. 
certainement d'être approfondi. Tant pour l'histoire de la chanson sentimentale européenne que dans le contexte nord-américain, où l'impact des États-Unis semble toujours laminer la compréhension des modes et des standards, cette filière italienne gagnerait à faire l'objet de travaux, notamment pour reconstituer les réseaux d'interlocuteurs culturels et industriels qui rendent possible cette circulation directe et indirecte entre la chanson populaire italienne et ses cousines des années 1930 et 1940 .

\section{Autres transits culturels}

Outre la question des transferts culturels sur le plan géographique (Québec, France, États-Unis, Italie) et sur celui des supports (scène, partitions, disques, films, magazines), notre analyse de quelques étapes de la circulation de J'attendrai incite à considérer d'autres aires de transit culturel qui façonnent le succès dans le domaine de la chanson populaire. J'attendrai témoigne, par exemple, d'une circulation à différents " étages " de la hiérarchie culturelle, et nous offre ainsi un cas exemplaire des échanges et des emprunts qui nourrissent les sphères culturelles restreinte et de grande consommation, pour reprendre le cadre théorique de Pierre Bourdieu $^{28}$. Une chanson à succès témoigne ainsi parfois d'un processus de migration entre des registres légitimes, comme celui du monde de l'opéra, et une chanson populaire relevant des variétés, dont le succès auprès du grand public s'accompagne d'un discours dénigrant de la part des principaux critiques culturels et musicaux. L'ancrage de J'attendrai dans la musique plus savante par l'emprunt d'une partie d'un air de l'opéra Madame Butterfly de Puccini permet de constater que des airs, des pratiques, des personnages, un éthos circulent entre des champs musicaux et culturels que les critiques et les analyses tendent à opposer. La variété des versions québécoises de J'attendrai donne à penser que même à l'échelle locale, cette porosité

28 Pierre Bourdieu, Les règles de l'art : genèse et structure du champ littéraire, Paris, Seuil, 1992. 
des registres de légitimité est bien présente. En témoignent, par exemple, la version de Ludovic Huot, membre du Trio lyrique et surtout reconnu dans ce contexte, dont le style vocal est issu de la formation classique (techniques du bel canto), et la version de Jean Lalonde, chanteur de charme surnommé «le Dom Juan de la chanson ", qui exploite la palette d'interprétation plus typique du crooner, un registre moins étendu, une technique personnelle davantage ancrée dans la parole parlée quotidienne que soumise aux codes d'une formation musicale traditionnelle ${ }^{29}$.

L'autre aire de circulation qui mérite considération est assurément la question des rapports sociaux entre les sexes qu'implique une chanson, tant du point de vue de l'énonciation ${ }^{30}$ que de la réception. Dans le cas de J'attendrai comme dans ceux de quelques autres chansons figurant à notre palmarès des préférences musicales, nous avons constaté, pour le début des années 1940 surtout, que plusieurs chansons pouvaient, avec ou sans transformations (pronoms, féminisation de certains mots, etc.), être interprétées par des hommes ou des femmes. Nous avons donc inclus cet aspect à notre palette d'observation des différents indicateurs du succès d'une chanson. Il est étonnant de constater, par exemple, qu'aucune interprète féminine québécoise avant Alys Robi n'enregistre de version locale de succès américains ou français nommés par nos lectrices du Bulletin des agriculteurs dans les années 1940, même si plusieurs versions françaises

29 «À l'inverse [de l'opéra], les chanteurs populaires entrent dans leur art par une initiation spontanée, par la famille, le groupe communautaire, les copains, la rue... Le chanteur populaire est le plus souvent un autodidacte. [...] Parmi eux, beaucoup travaillent aussi inlassablement le chant, mais leur voix, orientée sur des registres plus restreints et des timbres non lissés, est plus sauvage, plus brute, plus insoumise, moins codifiée " (Catherine Dutheil-Pessin, La chanson réaliste : sociologie d'un genre, Paris, L'Harmattan, 2004, p. 56).

30 Au sujet des particularités de la chanson sur le plan de l'énonciation et, plus particulièrement, concernant le fait que les auditeurs sont parfois les destinataires, mais deviennent souvent, ne serait-ce qu'en fredonnant les chansons, les énonciateurs, voir Vincent Delecroix, Chanter : reprendre la parole, Paris, Flammarion, 2012. 
et américaines des chansons sont interprétées par une ou des femmes. Les transferts culturels semblent donc parfois avoir un sexe, et les reprises québécoises sont toutes enregistrées par des hommes au Québec, même si les succès peuvent, ailleurs, être portés par des femmes.

Or, tout particulièrement pour la chanson sentimentale, l'inversion des modèles culturels masculins et féminins dans la relation amoureuse qu'implique la reprise par des hommes de chansons conçues d'abord pour des femmes, et les conséquences de cette inversion sur les représentations traditionnelles du masculin et du féminin ne représentent pas les seuls aspects de la chanson qui se trouvent transformés. Ce jeu sur le sexe de l'interprète se révèle, en effet, assez commode pour favoriser un double rôle du public et, tout particulièrement, des femmes, qui se trouvent ainsi, simultanément destinataires et destinatrices des chansons, qu'elles les fredonnent réellement ou non. Cette dimension participative nous ramène elle aussi à une époque qui précède l'enregistrement et aux différentes pratiques auxquelles la chanson donnait lieu ${ }^{31}$. Elle nous rappelle de ce fait, tout particulièrement pour les phénomènes culturels populaires, et dans la mesure où la culture est, comme le souligne Michel de Certeau, "appropriation (ou réappropriation) ${ }^{32}$ ", que nos inventaires de ces pratiques et les interrogations qu'elles suscitent gagnent à concevoir le rapport des consommateurs aux œuvres comme une « manière de penser, investie dans une manière d'agir ${ }^{33}$ ".

31 Je pense ici à la façon dont les chansons circulent par le bouche à oreille et à l'ensemble des pratiques associées à l'oralité, et tout particulièrement au folklore. Voir à ce sujet les travaux de Luc Lacourcière sur la chanson populaire et ceux de Conrad Laforte sur la poétique de la chanson traditionnelle.

32 Michel de Certeau, L'invention du quotidien, t. 1 : Arts de faire, Paris, Gallimard, 1990, coll. «Folio essais ", p. XI.

33 Ibid., p. XLI. 


\section{Conclusion}

Le cas de la circulation de la chanson J'attendrai dans différents espaces et dans diverses aires culturelles est riche, et nous ne prétendons pas ici en avoir épuisé la substance, loin de là. Néanmoins, le matériel colligé nous permet un certain nombre de constats éclairants. Le premier concerne l'importance de la prise en compte de l'ensemble du continuum historique et de la circulation des œuvres. Si le poids culturel des grands centres que sont Paris, Londres ou New York pèse lourd dans l'embrayage et la canalisation des succès, les rapports qu'entretiennent les espaces culturels plus marginaux avec ces grands centres apparaissent comme des filières du succès moins conventionnelles, et les écarts que ces espaces de production locaux introduisent par rapport aux standards internationaux sont riches de détails et d'énigmes révélatrices. Ils mettent en lumière les négociations et les adaptations de cette musique circulant sur la scène globale à l'échelle des champs socioculturels locaux. Le second constat, découlant du premier, nous a mis sur la piste de certaines spécificités des versions québécoises d'une chanson à succès au sein du vaste ensemble des différentes versions de cette chanson.

Outre la circulation dans l'espace géographique, le cas de J'attendrai incite à inscrire la circulation de la chanson à l'ère de l'enregistrement sonore dans le sillage de la chanson de tradition orale et à nous inspirer, notamment, des types de travaux auxquels ce mode de transmission a pu donner lieu, surtout pour la période qu'on pourrait dire de transition entre les deux régimes, soit celle des années 1930 et 1940. Nous pensons ici, par exemple, aux travaux réalisés dans la foulée des études culturelles sur la circulation des chansons ${ }^{34}$ et des "song networks " ${ }^{35}$, ou au travail d'un Luc Lacourcière sur

${ }^{34}$ Steven Feld, « Pygmy POP: A Genealogy of Schizophonic Mimesis ", Yearbook for Traditional Music, vol. 28 (1996), p. 1-35.

35 Keir Keightley, "Un voyage via Barquinho: Global Circulation, Musical Hybridization, and Adult Modernity, 1961-9 ", dans Jason Toynbee et Byron Dueck, Migrating Music, New York, Routledge, 2011. 
Le Rapide-Blanc d'Oscar Thiffault ${ }^{36}$, qui montre bien la porosité des deux mondes musicaux qu'on distingue spontanément (transmission orale et musique enregistrée), au risque d'affirmer les choses les plus incongrues. L'abondance des versions favorise d'ailleurs une saisie semblable de l'objet, de même que le jeu des variantes pose des énigmes qui constituent autant de défis pour les chercheurs. La considération des versions multiples est, par ailleurs, tout particulièrement utile pour sonder les modalités d'adaptation d'une chanson à différents styles musicaux et, à cet égard, elle contribue à ajouter une dimension poétique aux considérations plus historiques que ce type d'étude appelle.

Toutefois, l'oralité à l'ère de la reproductibilité technique, pour reprendre l'expression de Walter Benjamin, s'est déplacée, et c'est dorénavant par la radio, le disque et le cinéma qu'elle circule. Si la question des transferts culturels doit être abordée pour la chanson populaire, elle implique nécessairement le recours à toutes les sources médiatiques qui permettent la circulation de la voix chantée, qu'il s'agisse de la scène (salles de concert, cabarets, music-halls) ou des médias électroniques (radio, disque). C'est bien l'ensemble de ce continuum qui contribue à remodeler l'oralité et à fixer de nouveaux codes de proximité (gros plan au cinéma et voix douce amplifiée pour la chanson). À la lumière de ces considérations, les transferts culturels ne se font pas que sur le plan géographique, mais doivent intégrer la question de la médiation afin de donner toute sa mesure à l'engouement du public pour des chansons, des figures populaires. En outre, la circulation géographique elle-même ne concerne pas uniquement les frontières géopolitiques des États, mais également la prise en compte du rapport entre les métropoles et les régions, variable tout particulièrement pertinente pour le palmarès que nous avons établi.

${ }^{36}$ Luc Larcourcière, "Les transformations d'une chanson folklorique : du Moine tremblant au Rapide-Blanc ", Essais sur Émile Nelligan et sur la chanson populaire, Montréal, Fides, 2009, p. 301-334. 
Au terme de ces considérations, il nous paraît important de prolonger la réflexion et de continuer à intégrer la question des transferts culturels aux études sur la chanson à succès. D'une part, cette approche se justifie en permettant de retracer l'histoire des différents relais qui assurent la naissance d'un phénomène. D’autre part, elle apparaît incontournable pour cerner les différentes strates de sens qui se relaient et se fixent, plus ou moins durablement, pour assurer la pérennité du phénomène. Ces deux éléments contribuent ainsi à dé-naturaliser autant qu'à dé-univocifier, si vous nous permettez le néologisme, la notion même de succès, d'une richesse souvent mésestimée. 